\title{
SOFTWARE
}

\section{Simulating the imaging process in scattering tissue}

\author{
Biobeam is a software package that simulates optical effects during imaging within scattering tissue, which could \\ help improve image acquisition and extraction.
}

maging within tissue is afflicted by light scattering and aberrations, which degrade image quality. One way to understand and potentially address these issues is to model these distortions. The understanding gained from such simulations could lead to approaches that avoid the degradation of image quality and improve the process of image restoration from suboptimal images. However, realistic simulations involve modeling of the point spread function at each point within the sample, which is computationally demanding.

To address this problem, Moritz Kreysing and his colleagues at the Max Planck Institute of Molecular Cell Biology and Genetics in Dresden have developed biobeam, a computationally efficient simulation tool that adapts the beampropagation model for fiber optics to biological samples. Their approach relies on multiplexing of the point spread function calculations and efficient parallelization of these computations, which will make realistic simulations easier and faster.

The researchers demonstrated biobeam in a simulation that mimicked imaging of a Drosophila embryo by light-sheet microscopy. They sent a light sheet through a simulated embryo and modeled the aberrations at each point within the embryo, on the basis of the refractive indices of nuclei, cytoplasm and organelles. This simulation provided them with an image closely resembling the images obtained in real experiments that recapitulated typical imaging artifacts such as blur, contrast loss and distortions, among others. The team further validated the approach in other imaging scenarios.

Biobeam can also be used to simulate the effects of adaptive optics. In adaptiveoptics-based imaging, crisp imaging foci within scattering media are generated by shaping of the wavefront that enters the sample such that aberrations are counteracted. Kreysing and his team were able to simulate this process and reproduce phenomena associated with adaptive optics such as, for example, the shift-shift memory effect, which explains the insensitivity of adaptive-optics-based imaging against lateral shifts of the optical focus.

Kreysing and his team propose that biobeam may facilitate testing approaches for improving image quality in large and scattering samples. Although the authors mainly demonstrated the approach for light-sheet microscopy, it is also applicable to other imaging modalities.

Nina Vogt

Published online: 31 May 2018

https://doi.org/10.1038/s41592-018-0031-1

\section{Research papers}

Weigert, M. et al. Biobeam-multiplexed wave-optical simulations of light-sheet microscopy. PLoS Comput. Biol. 14, e1006079 (2018).

\section{We Create Solutions}

Ultra Precise Motion Control - D.C. Servo motors down to $20 \mathrm{~nm}$, piezos down to $1 \mathrm{~nm}$, and low drift XYZ stages.

Microscopy - Automation, modular microscopes, autofocus complete light sheet systems, and components. OEM - Custom designed systems to user specifications.

\section{VISIT US AT:}

$3^{\text {rd }}$ EMBO Practical Course on Light Sheet Microscopy - Aug. $2^{\text {nd }}-11^{\text {th }}, 2018$ - Dresden, Germany $10^{\text {th }}$ Annual LSFM International Conference $\cdot$ Aug. $12^{\text {th }}-15^{\text {th }}, 2018 \cdot$ Dresden, Germany 